\title{
Modulating the Intestinal Microbiota: Therapeutic Opportunities in Liver Disease
}

\author{
Cyriac Abby Philips*1, Philip Augustine ${ }^{1}$, Praveen Kumar Yerol ${ }^{2}$, Ganesh Narayan Ramesh ${ }^{3}$, \\ Rizwan Ahamed ${ }^{1}$, Sasidharan Rajesh ${ }^{1}$, Tom George ${ }^{1}$ and Sandeep Kumbar ${ }^{1}$
}

${ }^{1}$ The Liver Unit, Monarch Liver Lab and Division of Gastroenterology, Cochin Gastroenterology Group, Ernakulam Medical Centre, Kochi, Kerala, India; ${ }^{2}$ Department of Gastroenterology, State Government Medical College, Thrissur, Kerala, India; ${ }^{3}$ Department of Gastroenterology, Aster Medcity, Kochi, Kerala, India

\begin{abstract}
Gut microbiota has been demonstrated to have a significant impact on the initiation, progression and development of complications associated with multiple liver diseases. Notably, nonalcoholic fatty liver diseases, including nonalcoholic steatohepatitis and cirrhosis, severe alcoholic hepatitis, primary sclerosing cholangitis and hepatic encephalopathy, have strong links to dysbiosis - or a pathobiological change in the microbiota. In this review, we provide clear and concise discussions on the human gut microbiota, methods of identifying gut microbiota and its functionality, liver diseases that are affected by the gut microbiota, including novel associations under research, and provide current evidence on the modulation of gut microbiota and its effects on specific liver disease conditions.

Citation of this article: Philips CA, Augustine $\mathrm{P}$, Yerol PK, Ramesh GN, Ahamed R, Rajesh S, et al. Modulating the intestinal microbiota: Therapeutic opportunities in liver disease. J Clin Transl Hepatol 2020;8(1):87-99. doi: 10.14218/ JCTH.2019.00035.
\end{abstract}

\section{Identification and study of the gut microbiota (GM)}

The microbiota is defined as all of the microbes associated with complex organisms such as humans, whereas the microbiome is the complete representation of these microbes and their genes. Initial methods to identify and characterize microbiota were predominantly based on culture techniques. High-throughput culturing, which combines controlled and automated cell-culturing systems to grow dozens of cultures at once over long periods, has essentially improved characterization of novel microbes and strains (also known as

Keywords: Microbiome; Metagenomics; NGS; Cirrhosis; Illumina.

Abbreviations: $A C L F$, acute-on-chronic liver failure; $A H$, alcoholic hepatitis; $A I H$ autoimmune hepatitis; ALD, alcoholic liver disease; APAP, acetaminophen; FMT, fecal microbiota transplantation; FXR, farnesoid-X receptor; GM, gut microbiota; $\mathrm{HCV}$, hepatitis C virus; HE, hepatic encephalopathy; IL, interleukin; LPS, lipopoIysaccharide; MELD, model for end-stage liver disease; NAFLD, nonalcoholic fatty liver disease; NASH, nonalcoholic steatohepatitis; PSC, primary sclerosing cholangitis; TGR5, G protein-coupled bile acid receptor 1 and membrane-type bile acid receptor; TNF, tumor necrosis factor.

Received: 11 August 2019; Revised: 11 October 2019; Accepted: 27 October 2019

*Correspondence to: Cyriac Abby Philips, The Liver Unit, Monarch Liver Lab and Division of Gastroenterology, Cochin Gastroenterology Group, Ernakulam Medical Centre, Kochi, Kerala 682028, India. Tel/Fax: +91-484-2907000, E-mail: abbyphilips@gmail.com culturomics). Apart from high-throughput culturing, the 'continuous culturing' utilizes an open system which is constantly supplied at one end with fresh growth medium and overflow allowed to drain from the vessel at the other end, diluting out toxic metabolites and dead cells and leading to a 'steadystate' of microbial activity that can be further studied.

These techniques have been overwhelmed with the advent of sequence-based approaches, in which, without the need for growing microbes in the lab, complete detail of the species present in the sample can be attained within a short time. Marker gene survey is the most common sequence-based approach used for microbial characterization. This method utilizes identification and comparison of the microbiome in the sample with universal marker genes, thereby identifying all known species within the sample based on unique conserved DNA. The universal marker gene that is most widely employed is the small subunit ribosomal RNA gene (16s rRNA gene for bacteria and archaea, 18s rRNA gene for eukaryotes).

Using the standard PCR technique, en masse sequencing of the extracted DNA is performed, and resulting data is clustered by comparative sequence similarity into Operational Taxonomic Units that are mapped against a comprehensive reference database to assign taxonomic classifications - thereby, representing an approximation of species within the sample. Whole-genome sequencing employs sequencing of multiple microbial genomes in a single run, by multiplexing samples through the addition of unique sequence tags. Shotgunsequencing - in which extracted DNA is randomly fragmented before sequencing and then the resulting overlapping sequence data combined bioinformatically by mapping onto an existing reference genome into continuous stretches - is the standard method of whole-genome sequencing. Wholegenome-sequencing typically requires that the organism be grown in culture first before DNA extraction and sequencing.

With metagenomics, direct sequencing is performed on DNA extracted from a sample, followed by bioinformatical piecing together of the sequenced data into continuous data, allowing for study of the qualitative as well as functional aspect of the microbiota without culturing. Single-cell genomics utilizes isolation of individual microbes from the sample, after which whole genome amplification is performed. This powerful technique allows for recovery of genomic data of rare species and helps in the understanding of organisms that are capable of carrying out a particular metabolic function, even if such genomic and functional information is missing from comparative databases.

Metatranscriptomics, or RNA sequencing, in contrast to metagenomics, provides detailed information on the functional 
activity of the microbiota at a given time and under prevailing environmental conditions, and not only the functional potential as seen with the latter. In metaproteomics, whole characterization of the entire microbiota protein complements at a given point in time is analyzed, which provides information that can be linked to species-based information. This enables the direct study of translated genes, revealing important metabolic information of the microbial community. Metabolomics provides metabolic profiling with regards to functional pathways of a given organism or groups in a given sample (Supplementary Fig. 1). A simplified summary of various mechanisms for identifying and studying GM is shown in Fig. $1 .^{1-4}$

\section{Introduction to GM}

The Metagenomics of the Human Intestinal Tract (referred to as MetaHIT) and the Human Microbiome Project provided full detail of the human-associated microbial repository. The data classified bacterial communities into 12 different phyla, of which $93.5 \%$ belonged to Proteobacteria, Firmicutes (grampositives), Actinobacteria and Bacteroidetes (gram-negative anaerobes). In the early stages of human development, the microbiota has low diversity, dominated by Actinobacteria and Proteobacteria. The main genera among Bacteriodetes in the gut include Bacteroides and Prevotella, while the major Firmicutes genera are Clostridium, Blautia, Enterococcus, Faecalibacterium, Eubacterium, Roseburium, Ruminococcus, Streptococcus and Lactobacillus. Actinobacteria mainly consist of Bifidobacteria, Atopobium and Collinsella, while Proteobacteria consist of Enterobacteriaceae like the Escherichia and Klebsiella. Verrucomicrobia is represented by only one species in humans, Akkermansia muciniphila. Apart from these, the kingdom Archaea, predominated by Methanobrevibacter species and Eukarya, such as Candida, are also present. Lower representations by parasites, viruses and bacteriophages are also notable (Supplementary Fig. 2).

At around 3 years of age, the composition, diversity and functionality of a child's microbiota resemble that of an adult. Above 65 years of age, higher Bacteroidetes phyla and Clostridium cluster IV occur. It is important to note that the GM of an adult is in a state of constant remodeling based on sex, genetic make-up (the gut microbiome is heritable), environmental, dietary, habitual, objective and subjective interactions, and acute and chronic disease conditions as well as spatial differences within the subject. For example, the intestinal luminal and mucosal microbiota compositions are significantly different in the same person, while the intestinal luminal microbiota differs from person to person, from region to region, and between sexes. Normally, the abundance of Bacteroidetes is higher in luminal (fecal) samples than in the intestinal mucosa, in which the Firmicutes, specifically Clostridium cluster XIVa, is higher. The theory of 'core microbiota' - that is a fixed set of groups of organisms present in all individuals - was proposed, but recent observations have shown that the commonness lies at the level of the microbiome (and not microbiota), suggestive of a 'functional core microbiota', which remains to be wholly defined in healthy persons..$^{5-11}$

\section{The functional microbiota}

The gut metabolome is mostly derived from carbohydrate, protein, and lipid metabolism. The major metabolites

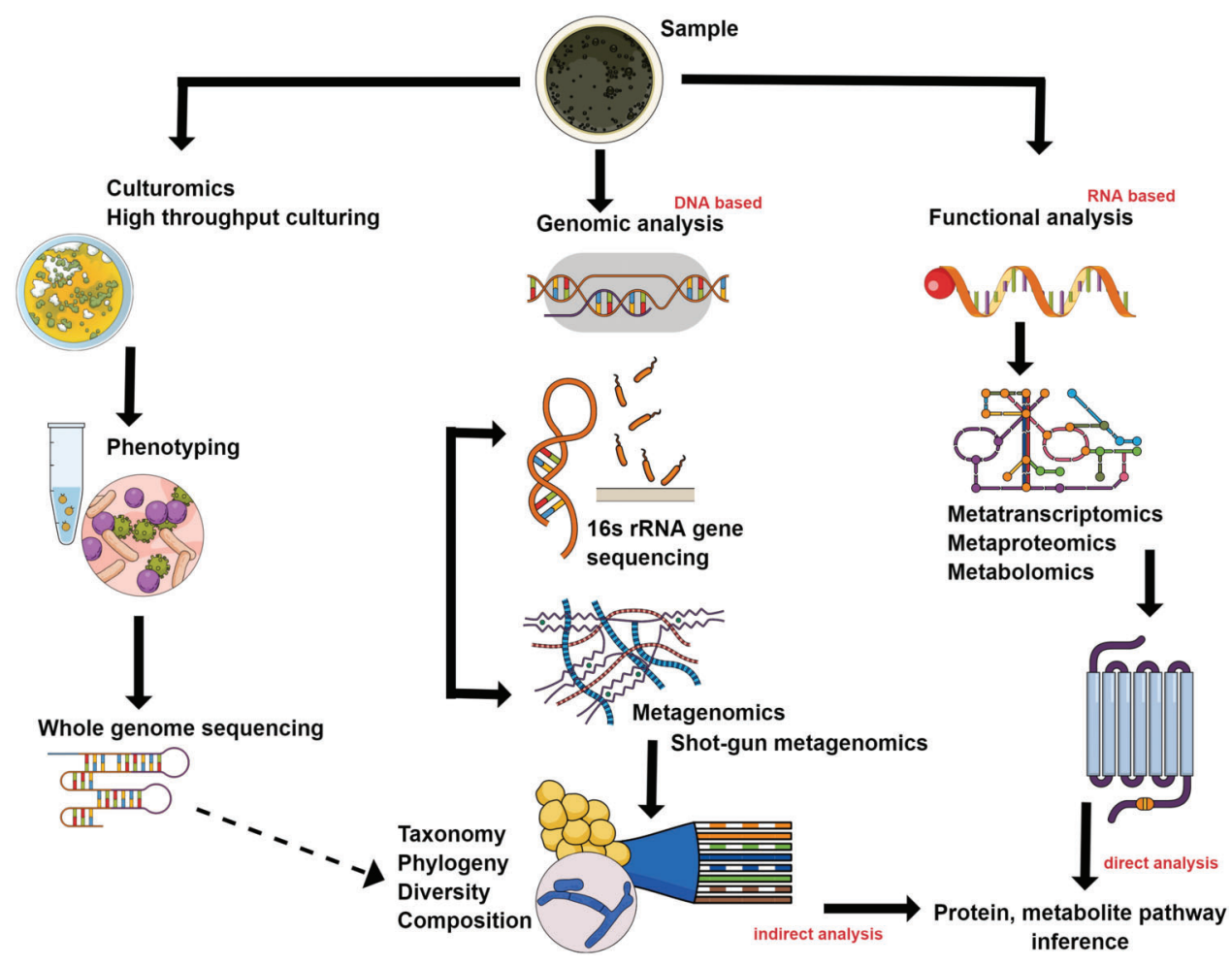

Fig. 1. Various methods for identifying and studying the gut microbiota and microbiome. Culturomics help in phenotyping the microbial communities, and further whole genome sequencing improves identification of microbial diversity up to the species and functional levels. DNA-based genomic analysis through $16 \mathrm{~s}$ rRNA sequencing curtails time to identification of microbial species, while RNA-based analysis helps in studying microbial function at the transcriptome, proteome and metabolite levels. 
produced in the gut include short-chain fatty acids, branchedchain fatty acids, branched-chain amino acids, biogenic amines, organic gases, and other secondary plant-derived compounds, such as choline, bacterial cell wall components, polyamines, and volatile organic compounds. The plethora of metabolites thus formed are further absorbed, distributed or excreted by multiple, highly dynamic processes and pathways that significantly affect human health by regulating pro- and anti-inflammatory processes, immunological landscape (innate and adaptive immunity), and detoxification. For maintaining homeostasis, the intestinal barrier limits exposure of the host immune system to the microbiota through multifactorial and dynamic processes that operate at the luminal and mucosal level. ${ }^{12,13}$

The GM caters to and provides multiple benefits to the host, such as nutrient metabolism and assimilation, protection and control over pathogenic species, and maintenance of immune functions. For example, the colonic bacteria express carbohydrate-active enzymes, which empower them to ferment complex carbohydrates, thereby generating metabolites which regulate cellular processes such as gene expression, chemotaxis, differentiation, proliferation, and apoptosis. On the other hand, specific anaerobes produce acetate while propionate and butyrate are produced by different subsets of gut bacteria through distinct molecular pathways. In the human intestine, propionate is mainly produced by Bacteroidetes, while the production of butyrate is mainly by Firmicutes. Starch fermentation by Eubacterium rectale or Eubacterium hallii that belong to Firmicutes, significantly contributes to butyrate production in the colon and Akkermensia muciniphila has been found to majorly contribute to propionate production through mucin degradation, the latter which is primarily absorbed by the liver. Propionate has been shown to reduce cancer cell proliferation and through its action on beta-cell function, ameliorates reward-based eating behavior though striatal pathways. In addition, butyrate is known for its anti-inflammatory activities in the liver microenvironment, acting by attenuating bacterial translocation and enhancing gut barrier strength by improving tight-junction function. Similarly, the short-chain fatty acids produced by the colonic GM regulate the immune system and inflammatory processes by influencing the production of interleukin (IL)-18, which is involved in maintenance and repair of mucosal epithelial integrity as well as in modulation of appetite regulation and energy utilization in the host, which are associated with metabolic syndrome and obesity.

Apart from carbohydrate metabolism, pertinent lipid metabolism in the host is also driven by the GM. For example, the facultative and anaerobic bacteria of the colon produce secondary bile acids which enter the systemic circulation to modulate hepatic and systemic lipid metabolism through nuclear or $\mathrm{G}$ protein-coupled receptors. Akkermansia, Christensenellaceae, Tenericutes, Eggerthella, Pasteurellaceae, and Butyricimonas are associated with body mass index in patients with metabolic syndrome as well as levels of triglycerides and high-density lipoproteins. ${ }^{14-18}$ With regards to protein metabolism, the microbiota-derived metabolites produced from aromatic amino acids (tyrosine, tryptophan, and phenylalanine) affect host signaling pathways interacting with host immunity. Bacteroides thetaiotaomicron, Proteus vulgaris, and Escherichia coli act through tryptophanase activity, producing indole which is sulfated in the liver and resulting in the production of 3-indoxyl sulfate and related compounds which promote systemic inflammation through transcription of IL-6.

Indole-3-propionate acts at the pregnane $X$ receptor (referred to as PXR) and down-regulates tumor necrosis factor (TNF)-alpha production in enterocytes by limiting bacterial translocation and lipopolysaccharide (LPS) infiltration into the circulation, thereby reducing metabolic endotoxemia and host inflammation. ${ }^{19-22}$ Various microbes or groups of microbes are associated with carrying out specific regulatory processes in the human gut, which is directly or indirectly associated with liver health (Supplementary Fig. 3).

\section{Microbiota and the gut-liver-axis}

Since the liver is an organ that has privilege in placement with regards to maximal exposure to gut microbes and its metabolites, studies on 'healthy state' and diseases associated with the hepatobiliary system have been on the forefront in the current bench-to-bedside research. Changes associated with the GM are implicated in the pathogenesis of many liver diseases. This alteration in general is termed dysbiosis, in which there is an imbalance between the symbionts and pathobionts in the gut. The intestine and liver have a bidirectional communication mediated through the biliary tract, portal vein, and systemic circulation. The liver communicates with the gut through bile acids and other metabolic mediators. In the gut, the microbes metabolize endogenous and exogenous compounds, end-products of which translocate to the liver through the portal vein, influencing the liver microenvironment and functions. The liver receives and filters large amounts of nutrients, bacterial products, toxins and metabolites through the portal vein, with an efferent circulation via the biliary system. This 'metabolic endotoxemia', as described by Cani et $a .^{23}$ in patients with diabetes and metabolic syndrome promotes a steady-state of low-grade inflammation within the liver microenvironment, driven by unhealthy changes in the GM.

Similarly, a 'tip of the balance' towards a more pathogenic profile of microbiota leads to increased exposure of the liver to pathogen and microbe-associated molecular patterns through an increase in bacterial translocation and leakiness of the gut. This exposure results in proinflammatory cellular signaling within the hepatic environment driven by major cytokines, such as IL-1, IL- 6 and TNF-alpha. Continuous proinflammatory state, in the presence of persistence of factors that promote it (such as alcohol, drugs, obesity, diabetes) leads to production of reactive oxygen species which promote liver injury and fibrosis.

Liver, a highly active site of metabolism and immune homeostasis, handles and secretes multiple immunogenic molecules and metabolites into the gut, which affects the microbiota and vice-versa. Secretory immunoglobulin $A$, bile acids and fatty acids processed by the liver activate various nuclear receptors, such as the G-protein coupled receptor and farnesoid-X receptor (FXR), that regulate glucose and lipid metabolism and homeostasis as well as conjugation and detoxification of exogenous and endogenous toxins. Gutderived hormones (for example, fibroblast growth factor, glucagon-like-peptide-1 and serotonin) also play an important role in maintaining homeostasis and energy assimilation and balance by affecting the steady metabolic state, via their action on appetite and food intake. Dysbiosis has been shown to initiate, promote or cause progression of liver diseases, such as alcoholic liver disease (ALD), alcoholic hepatitis (AH), 
nonalcoholic fatty liver disease (NAFLD) and nonalcoholic steatohepatitis (NASH), drug-induced toxic liver injury, liver fibrosis, cirrhosis and its complications, hepatocellular carcinoma, and chronic cholestatic and autoimmune hepatobiliary disease (Fig. 2). ${ }^{24-27}$

\section{GM and diseases of the liver}

\section{NAFLD and NASH}

NAFLD encompasses steatosis, steatohepatitis, advanced fibrosis, cirrhosis and related hepatocellular carcinoma. High-quality studies in humans with and without NAFLD/ NASH have shown a strong correlation of GM in the initiation
Philips C.A. et al: Intestinal microbiota reinstitution therapy

and progression of NAFLD-associated conditions. ${ }^{28,29}$ Lower levels of Bacteroides are associated with obese patients with $\mathrm{NASH}$, while the lower abundance of Firmicutes was demonstrated in non-obese NASH patients. Reduction in Lachnospira, Ruminococcus and Lactobacillus was notable in lean patients with NASH. In adolescents, the abundance of Bacteroides followed a ' $U$ ' pattern, based on the dietary pattern of fat intake. In those with high fat intake, low and high abundances were noted, while in those with low fat intake, a moderate level of abundance was found.

Bilophila, Paraprevotella and Suturella are associated with higher hepatic fat content, in contrast to Oscillospira and Varibaculum for which a negative association with steatosis has been demonstrated. Higher levels of Bilophila wadworthia

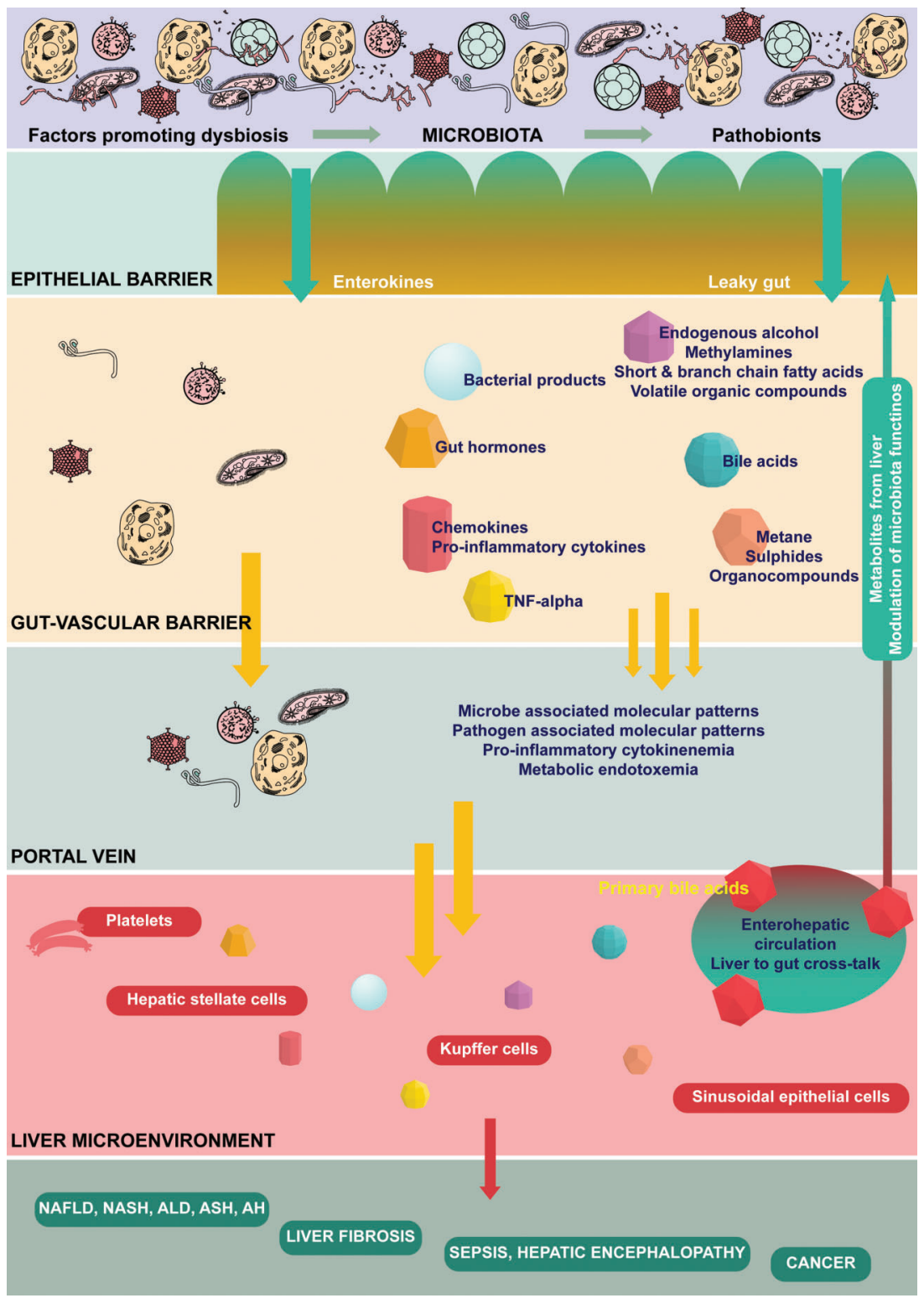

Fig. 2. The gut-liver axis and microbiota related cross-talk. In the presence of factors that disrupt microbial diversity and function (alcohol, metabolic diseases, drugs, environmental toxins, diet), pathobionts that promote disease causation or progression evolve in the dysbiotic milieu. This leads to gut barrier disruption, enhancement of local proinflammatory cascade, endotoxemia and ultimately systemic inflammation, leading to end organ adverse events. 
were found to be associated with $T$ helper-1-mediated gut inflammation. Lower levels of Oscillospira were associated with an increase in the metabolite 2-butanone, which was related to the onset of NAFLD. Increased levels of Ruminococcus, Dorea, Robinsoniella and Roseburia were found to be associated with progression of inflammation and fibrosis in NASH. Patients with NASH fibrosis $\geq 2$ were found to have higher abundance of Bacteroides and Ruminococcus, and lower levels of Prevotella; while, in those with advanced fibrosis and cirrhosis, Proteobacteria were relatively higher. At the species level, it was shown that Eubacterium rectale and Bacteroides vulgaris were relatively abundant in patients with mild to moderate NASH, while Bacteroides vulgatus and E. coli were predominant among patients with NASH-related advanced fibrosis and cirrhosis. ${ }^{30-32}$

Changes in GM in NAFLD patients have been shown to have positive correlation with small-intestinal bacterial overgrowth, defined as total bacteria growth of more than $10^{3}$ colony-forming-units of coliform bacteria per milliliter of proximal jejunal fluid, which is directly related to endotoxemia, circulating bacterial DNA and leakiness of the gut, and associated with worsening of steatosis and inflammation. Intestinal dysbiosis results in lower levels of colonic junctional adhesion molecule $A$ expression, increase in intestinal permeability, leading to liver exposure to higher levels of bacterial LPS, and endogenous ethanol, acetone and butanoic acid, that leads to hepatic inflammation. The GM also modulates choline metabolism, in which dietary choline is converted to dimethylamine and trimethylamine - increased levels of which result in hepatic inflammation. Lower levels of dietary choline lead to changes in GM and its functionality, which promotes fatty liver disease which, however, can reverse with high choline diet in small animal models. Escherichia, which increases production of endogenous ethanol utilizing the 'mixed-acid-fermentation' pathway was shown to be significantly associated with NASH and higher grades of fibrosis. The end metabolites that form out of ethanol degradation include acetate, which takes part in fatty acid synthesis, and acetaldehyde, which promotes cytotoxic effects within the liver microenvironment. Gut dysbiosis and small-intestinal bacterial overgrowth in NASH patients have been shown to be associated with higher levels of circulating serum TNFalpha and IL-8, through signaling mediated by toll-like receptors -9 and -4 .

Bile acids have significant effect on GM and have great impact on the metabolism of bile acids. Taurine- and glycineconjugated primary and secondary bile acids were found to be higher in patients with NASH compared to healthy controls. Bile acids, through regulatory effects on FXR and G proteincoupled bile acid receptor 1 and membrane-type bile acid receptor (referred to as TGR5), affecting the natural history of NAFLD. Agonists of FXR and TGR5 have been shown to reduce liver fat, improve NASH histology, and promote weight loss in a small animal model of NAFLD. ${ }^{33-35}$

Intestinal bacteria have been shown to reduce expression of fasting-induced adipocyte factor on the enterocytes, leading to induction of lipoprotein lipase activity and accumulation of hepatic triglycerides. Gut bacteria also promote increased production of short-chain fatty acids, acetate, propionate and butyrate, contributing to obesity, metabolic disease and increase in liver steatosis. LPS production by specific microbiota groups has been shown to promote liver carcinogenesis in mouse models, while in the germ-free mouse model, hepatocarcinogenesis was reduced. The occurrence of liver cancer in obese mouse models has been linked to the persistence of low-grade systemic inflammation that is initiated from dysbiotic microbiota. Thus, there is robust evidence that GM plays a central role in steatosis, inflammation and progression of fibrosis in NAFLD patients.

GM targeted therapies are upcoming strategies for treatment of NAFLD, NASH and NASH-related HCC. Currently, the focus of such treatments has been solely on probiotics and prebiotic supplementation - most commonly utilizing Lactobacillus and Bifidobacterium. Administration of probiotics has been shown to reduce liver enzymes, total cholesterol, TNFalpha, serum endotoxin levels, liver fat and NASH activity index in small-animal as well as human models. Probiotics increase PPAR-alpha activity and reduce metalloproteinases 2 and 9 and cyclooxygenase expression. Lactobacillus casei strain Shirota reduced the development of NASH in methionine- and choline-deficient diet mouse models by lowering serum LPS concentration; whereas Bifidobacteria and Lactobacillus rhamnosus GG ameliorated liver steatosis by acting on the sirtuin-1-mediated signaling pathway and reducing nuclear factor-кB inhibitor protein expression. ${ }^{36-38}$ Metaanalysis of randomized controlled trials on probiotics and prebiotics have shown that supplementation led to reduction in aminotransferase level, reduced body fat, and improved glucose metabolism. 34,39-41

Fecal microbiota transplantation (FMT), generally called 'stool transplant', utilized the transfer/transplantation of fecal microbiota from a healthy individual into a patient with dysbiosis, aiming to restore intestinal microbial diversity. There have been strong notions challenging the current terminology of FMT. The term 'fecal/stool' as a treatment modality has negative implications within the scientific community, pharmaceutical industry and funding sources as well as among patients and their families with regards to acceptance. Khoruts et al. ${ }^{42}$ proposed the term 'intestinal microbiota transplantation', while Bajaj et al. ${ }^{43}$ proposed that FMT be renamed 'microbiome restoration therapy'. However, both these terms feature inadequacies. Even though considered by some as 'an organ', the fecal microbiota is not an organ and is a highly different, unexplored entity, which differs in accordance with sex and region, and even between individuals. Hence, the term transplantation is inaccurate. We are yet to define a 'matching/healthy' microbiota donor. Microbiome, as discussed, is the genetic totality associated with the microbiota, which remains unique to the person. Studies have mostly looked at bacterial communities, even though eukaryotes, protozoa, viruses and phages are also part of the restoration, and which remain undefined. Hence, the term microbiome becomes vaguely general and does not hold well as a replacement to current terminology of FMT.

The term Microbiota Restoration Therapy ${ }^{\mathrm{TM}}$ has been patented by Rebiotix Inc (Ferring, Saint-Prex, Switzerland; www.rebiotix.com) to define their microbiota-based therapies. In this regard, an ideal, general, novel terminology for FMT, for utilization in discussions and in trials, in the light of current studies, would be 'intestinal microbiota reinstitution therapy', which can be modified accordingly, to further characterize different sites and specific components of the microbiota in future studies. We propose 'reinstitution' and not 'restoration' because the latter means 'to bring something back to the original form/former position or condition', when in reality, we are unaware of 'original' condition of microbiota within the recipient prior to the disease state; moreover, FMT actually modifies the microbiota more towards the donor 
profile and is not technically a 'restoration'. 'Reinstitution' means the act of establishing something again - and with FMT, we aim to establish a healthy microbiota. Studies on FMT in animal models have demonstrated amelioration of steatohepatitis in high-fat diet mice. ${ }^{44}$ Utilization of FMT for metabolic syndrome in humans was first performed by Vrieze et al. ${ }^{45}$ They found that FMT from lean donors temporarily increased peripheral insulin sensitivity and reduced hepatic steatosis without statistical significance. Xue et al. ${ }^{46}$ presented preliminary data on the effects of FMT $(200 \mathrm{~mL} /$ day for 3 days) in human NAFLD. They showed that change in a fat-attenuated parameter, as measured by FibroTouch ${ }^{\mathrm{T}}$, was significantly lower after treatment with FMT compared with the control group. Clinical trials assessing the efficacy and safety of FMT for NASH (NCT02469272) and NASH-related cirrhosis (NCT02721264) are ongoing.

\section{ALD}

Continuous or binge alcohol use over long periods results in ALD, which comprises liver steatosis, $A H$, alcoholic cirrhosis and acute-on-chronic liver failure (ACLF). Characteristic changes in intestinal microbiota have been shown to predispose to severe forms of ALD. Dysbiosis is associated with $A H$ in animal models, which were reversed with healthy donor FMT. Similarly, progressive worsening of dysbiosis is associated with the progression of alcoholic cirrhosis and its complications. Severe $\mathrm{AH}$ was associated with the higher fecal abundance of Bifidobacteria, Streptococci and Enterobacteria. Germ-free mice (C57BL/6) demonstrated higher susceptibility to alcohol-induced liver injury than conventional mice. ${ }^{47}$ This impresses the fact that complete absence of intestinal microbiota as well as an imbalance in microbiota both predispose to alcohol-related liver injury; hence, a 'eubiosis state' properly defines protection against alcohol-induced liver injury.

Defining the microbial communities that promote this eubiosis is still a matter of research. Change in GM has also been implicated in alcohol-induced damage to the liver through modulation of immune responses, expression of alcohol metabolism, oxidative stress, fat metabolism and endotoxemia. Alcohol use was shown to be associated with decreased levels of butyrate-producing Clostridiales and increased levels of pro-inflammatory Enterobacteriaceae and Proteobacteria. Lower abundance of Ruminococcus was associated with increased intestinal permeability and dysbiosis, which was reversed with abstinence. In $\mathrm{AH}$, reduction in the relative abundance of Clostridium leptum and Fecalibacterium prausnitzii has been demonstrated. ${ }^{46}$ Philips et al. ${ }^{48}$ demonstrated that patients with severe $\mathrm{AH}$ had a higher relative abundance of Enterobacter, Megaspaera, Dialister, Prevotella and Klebsiella, while in healthy controls, Akkermansia, Veillonella, Oscillopsira, Lachnospira, Bacteroides, Eggerthella, Coriobacterium and Bifidobacterium were higher. At the functional level, LPS biosynthesis, glycosyl transferase and valine-leucine-isoleucine degradation pathways were affected in patients with $\mathrm{AH}$, while in healthy controls, alanine-aspartate-glutamic acid metabolism and non-aromatic amino acid metabolism were significantly up-regulated. The gut microbiota composition in healthy and ALD and its effect on intestinal permeability in ALD pathogenesis point toward emerging evidence on GM modulation in ALD as a mode of treatment from preliminary clinical and non-clinical studies. ${ }^{49}$
Grander and colleagues ${ }^{50}$ showed that Akkermansia muciniphila abundance reduced with increasing severity of ALD and was lowest in $\mathrm{AH}$. Ciocan and colleagues ${ }^{51}$ showed that in patients with cirrhosis and $\mathrm{AH}$, total plasma bile acid levels were higher, while levels of total and secondary bile acids were lower compared to those without cirrhosis or $\mathrm{AH}$. The relative abundance of Actinobacteria was higher and that of Bacteroidetes which was lower in alcoholic cirrhosis with $\mathrm{AH}$. Puri et al. ${ }^{52}$ found that in alcohol-consuming patients, there was an enrichment of bacteria with genes related to methanogenesis and denitrification. Both heavy drinking controls and patients with severe $\mathrm{AH}$ demonstrated activation of a type III secretion system associated with gram-negative bacterial virulence. In patients with $\mathrm{AH}$ compared to non-alcohol consuming controls, there was an increase in isoprenoid synthesis through upregulation of the mevalonate and anthranilate degradation, which are known modulators of gram-positive bacterial growth and biofilm production, respectively. Bluemel et al. ${ }^{53}$ investigated the microbiota in the jejunum, ileum, cecum, feces and liver of mice subjected to chronic ethanol feeding; they found that chronic ethanol administration modified alpha diversity in the ileum and the liver, largely driven by an increase in gram-negative phyla, resulting in endotoxemia. Specifically, the gram-negative Prevotella increased in the mucus layer of the ileum and also in liver tissues, suggesting the central role of dysbiosis and bacterial translocation leading to liver injury with alcohol use.

In an open-label randomized controlled trial, probiotic-rich in Bifidobacterium bifidum and Lactobacillus plantarum, compared to placebo in patients with $\mathrm{AH}$, led to a reduction in hepatic inflammation in the form of improvement in liver biochemistry, while the addition of Lactobacillus casei Shirota 3 strain thrice daily for 30 days improved neutrophilic phagocytic capacity in ALD patients, when compared with baseline. ${ }^{54} \mathrm{~A}$ placebo-controlled trial showed that supplementation with $1.5 \mathrm{~g}$ of Bacillus subtilis and Enterococcus feacium daily for 7 days improved liver function and reduced systemic inflammation and endotoxemia in $\mathrm{AH} .{ }^{55}$ The first pilot study of FMT in steroid ineligible severe $\mathrm{AH}$ demonstrated an improvement in 1 -year survival in FMT-treated patients compared to historical controls ( $87.5 \%$ vs. $33.3 \%$ ). The relative abundance of Proteobacteria was high and that of Actinobacteria low in patients with severe $\mathrm{AH}$ at baseline. Post-FMT, this was significantly reversed, along with coexistence of protective symbiotic donor and recipient species at 12 months. Reduction in relative abundance of pathogenic species [Klebsiella pneumonia ( $10 \%$ to $<1 \%)$ ], and increase in non-pathogenic species [Enterococcus villorum ( $9 \%$ to $23 \%$ ) and Megasphaera elsdenii (10\% to $60 \%)$ ] was demonstrated. After FMT, reduction in methane metabolism, bacterial invasion of the epithelial cells, inflammatory and cytotoxic pathways, and aromatic amino acid generation was noted. ${ }^{48}$

In a retrospective observational study comparing FMT to other conventional modalities of treatment for $\mathrm{AH}$, the proportions of patients surviving at the end of 3 months in the steroids, nutrition, pentoxifylline, and FMT group were $38 \%$, $29 \%, 30 \%$, and $75 \%(p=0.036) .{ }^{56}$ In patients with severe $\mathrm{AH}$ and non-responders to steroids with ACLF grades 0,1 and $2+$ 3 , the 90 -day survival rates were $68.1 \%, 45.8 \%$ and $36.7 \%$. Philips and colleagues ${ }^{57}$ showed that, at the end of 548 days follow up, the proportion of ACLF-AH patients surviving, after FMT, in the lower (ACLF $0+1)$ and higher grade (ACLF $2+3$ ) groups were $72.7 \%$ and $58.3 \%$ respectively, which was higher than what is seen with current medical therapies and 
comparable to liver transplantation. Future studies on FMT in AH could identify better methods for fecal transfer, refine targeted therapy, and utilize precision metabolomics to modulate the intestinal milieu to improve outcomes.

\section{Role of GM in cirrhosis and its complications}

In liver cirrhosis, the presence of portal hypertension results in structural changes to the intestinal mucosa and vasculature, leading to an increase in intestinal permeability that worsens with gut microbial changes and associated functional metabolism. Altered microbiota has been found in the intestinal mucosa, stool and saliva samples from patients with cirrhosis of variable etiologies. The dysbiotic microbiota in patients with cirrhosis reveal a reduction in Bacteroides and Lachnospira and increase in Proteobacteria, Enterobacteria and Veillonella. The progressive increase in Enterobacteria correlates with complications of cirrhosis, especially hepatic encephalopathy (HE). The severity of cirrhosis with regards to Child-Pugh class correlated positively with Streptococcus and negatively with Lachnospiraceae (Coprococcus, Pseudobutyrivibrio, Roseburia). The decrease in autochthonous taxa such as Lachnospiraceae, Ruminococcaceae and Clostridiales XIV, and the relative increase in Staphylococcaceae, Enterococcaceae and Enterobacteriaceae were found to be associated with progressive liver failure and endotoxemia. The cirrhosis to dysbiosis ratio, between indigenous and non-indigenous taxa, negatively correlated with endotoxemia, was highest among healthy controls and lowest in patients with decompensated cirrhosis. A higher proportion of bacteria of buccal origin (Streptococcus and Veillonella) within the gut microbiome of patients with cirrhosis suggested that the oral microbiota invaded the gut, thereby contributing to the progression of the disease. Composition of the microbiota differed between patients with and without $\mathrm{HE}$, only in mucosa but not in stool samples. Veillonella, Megasphaera, Bifidobacterium and Enterococcus were prevalent in $\mathrm{HE}$, whereas Roseburia was more abundant in the non-HE groups. Minimal HE was associated with higher levels of Streptococcus salivarius, while in overt $\mathrm{HE}$, fecal levels of Alcaligenaceae and Porphyromonadaceae were associated with poor cognition. Salivary dysbiosis was greater in patients with cirrhosis who developed 90-day hospitalizations. Stool Bacteroidaceaeae and Clostridiales XIV predicted 90-day hospitalizations independent of such clinical predictors as Child-Pugh class and model for end-stage liver disease (commonly known as MELD) score. ${ }^{58-60}$

Bajaj et al. ${ }^{61}$ demonstrated distinct gut microbial profiles associated with ACLF in hospitalized patients. The cirrhosisto-dysbiosis ratio was lower in those with ACLF and also those with renal failure. Enterobacteriaceae, Campylobacteriaceae, Pasteurellaceae, Enterococcaceae and Streptococcaceae were associated with the development of poor outcomes, while Lachnospiraceae and Clostridiales were associated with a reduction in poor outcomes. Changes in the microbiota and dysbiosis had an independent and significant association with extrahepatic organ failure, intensive unit admissions, $A C L F$, and death in cirrhosis patients in-hospital.

In a randomized controlled trial, rectal enema-based FMT improved cognition among cirrhotic patients with recurrent $\mathrm{HE}$, significantly higher than seen in the control group. The MELD score transiently worsened post-antibiotics but reverted to baseline after FMT. Antibiotic therapy in the control group reduced beneficial taxa and decreased microbial diversity concurrent with Proteobacteria expansion, which was again reversed towards a beneficial pattern after FMT, leading to stable liver disease severity scores. The same group studied the utility of FMT capsules in patients with recurrent HE. In this phase 1 study, they found that oral capsule-based FMT treatment was safe and well-tolerated in patients with cirrhosis and recurrent $\mathrm{HE}$ and was associated with improved duodenal mucosal alpha diversity, reduced dysbiosis, antimicrobial peptide expression, reduced LPS binding protein level and improved EncephalApp performance. ${ }^{62,63}$

Even though circulating bacterial DNA, plasma endotoxin levels, and inflammatory and vasoactive markers in ascites and blood have been linked to infections in cirrhosis, especially spontaneous bacterial peritonitis, no direct linkage to dysbiosis or specific patterns of bacterial community changes have been studied.

\section{GM in liver cancer}

High-quality studies concerning experimental animal models supporting the role of GM changes and hepatocarcinogenesis are well known in the literature. In earlier studies, gut sterilization by antimicrobials in carcinoma animal models was shown to reduce tumor incidence and growth. Helicobacter hepaticus co-administration in the AFB-1 model of hepatocarcinogenesis revealed greater tumor number and size compared to AFB-1 alone. Chronic administration of diethylnitrosamine decreased the abundance of Lactobacillus, Enterococcus and Bifidobacterium species, leading to the promotion of tumor development and growth, which was then arrested by probiotic supplementation. LPS administration also resulted in increased number and size of HCC in animal models, which was attenuated via antibody and antimicrobial use. High-fat diet-related increase in gut dysbiosis and subsequent increase in deoxycholic acid resulted in hepatic tumor formation, which decreased with antibiotic treatment. Higher abundance of Atopobium, Bacteroides vulgatus, Bacteroides acidifaciens, Bacteroides uniforms, Clostridium cocleatum, Clostridium xylanolyticum and Desulfovibrio spp in a NASH mouse model was found to be associated with HCC development, which was again associated with a change in bile acid fractions in the liver tissue and plasma. The number and size of tumors was ameliorated using cholestyramine in the small animal model of NASH-related HCC. Prevotella and Oscilibacter, that are producers of anti-inflammatory metabolites, were found to be negatively associated with liver tumor formation. Studies linking dysbiosis and specific bacterial communities in human HCC is lacking, but targeting GM and its metabolites in patients with chronic liver disease is an exciting frontier for HCC management in the future. ${ }^{64,65}$

\section{Role of GM in other liver diseases - emerging indications}

Studies have shown that the intestinal microbiome could affect the development of autoimmune hepatitis (AIH) in predisposed individuals. A decrease in fecal Bifidobacterium and Lactobacillus abundance along with an increase in plasma LPS was notable in patients with higher severity of AIH. ${ }^{66}$ In germ-free mice, protection against experimental AIH was notable, in the presence of lower levels of leukocyte infiltration and inflammatory cytokines and absence of hepatocyte apoptosis due to the deficiency in activation of natural killer T cells in the liver microenvironment, that predisposes to autoantibody-mediated liver injury. Humans studies on the 
Philips C.A. et al: Intestinal microbiota reinstitution therapy

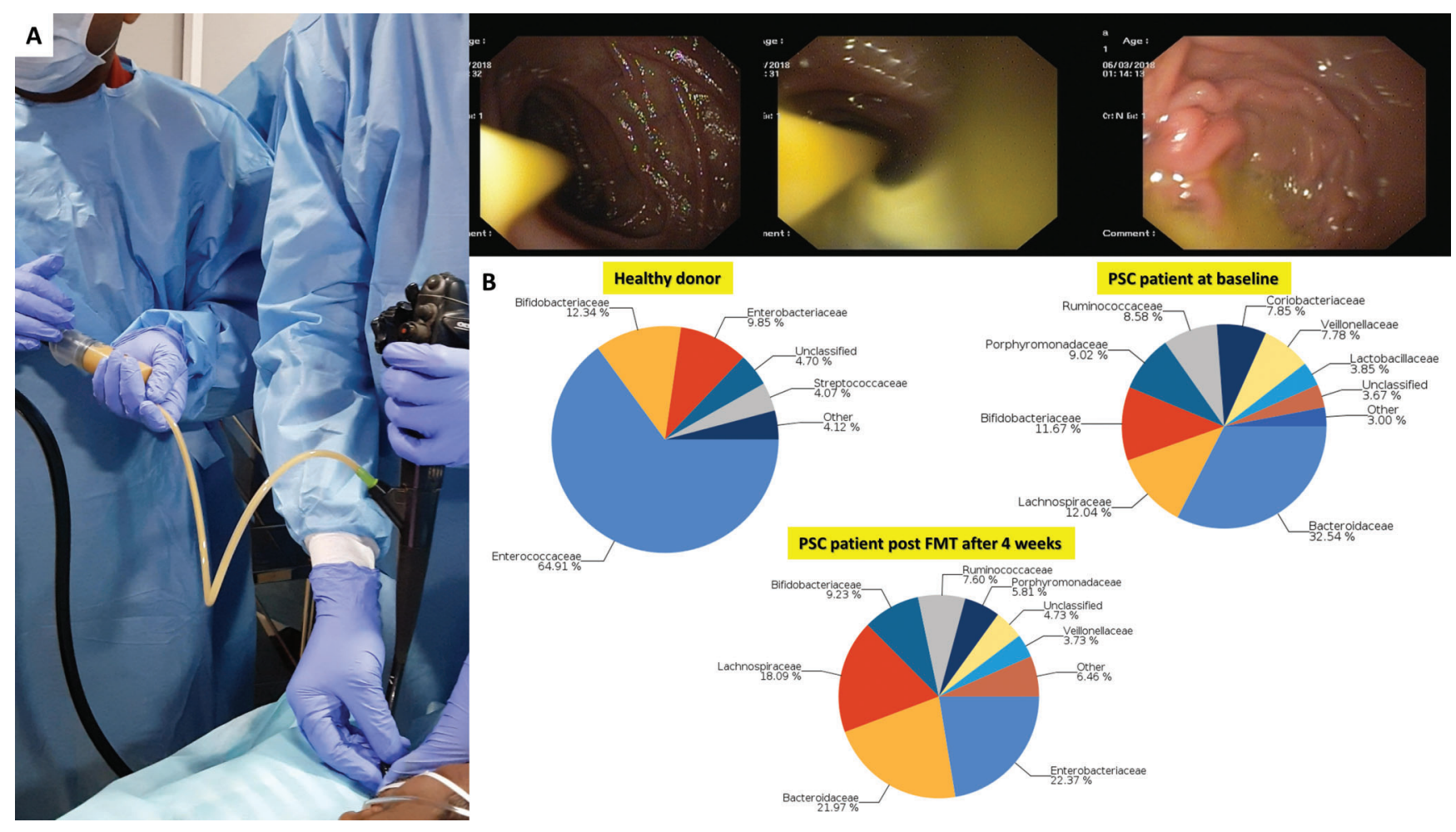

Fig. 3. Healthy donor microbiota restitution therapy through upper gastrointestinal endoscopy. Given to a patient with primary sclerosing cholangitis (A). The bacterial communities at the family level in the donor, the patient and the patient after 4 weeks (B). The modification of gut bacterial communities is evident, associated with improved clinical outcomes.

microbiome in AIH are lacking. Wei et al. ${ }^{67}$ showed that the gut microbiome of steroid treatment-naïve AIH had lower alphadiversity with distinct overall microbial composition when compared with healthy controls. Reduction in obligate anaerobes and increase in pathobionts such as Veillonella was associated with AIH - of which, Veillonella dispar was the most significantly disease-associated taxa with positive correlation with the elevation of aspartate aminotransferase. Thus, microbiota-based biomarkers could help identify AIH disease severity as well as being potential therapeutic targets.

GM-driven pathophysiological progression of primary sclerosing cholangitis (PSC) and primary biliary cholangitis are well documented in animal models. Bile acid metabolism is heavily handled by the GM and is central for the pathogenesis of PSC and primary biliary cholangitis. Functional gut microbial activities associated with bile acid metabolisms, such as dehydrogenation, conjugation and deconjugation and degradation of primary and secondary bile acids, and subsequent metabolite and toxin generation play an important role in autoimmune-mediated cholestatic inflammatory disease. Germ-free mice were found to have severe PSC features, in comparison to conventional mice, as the complete lack of microbiota resulted in alteration of needful bile acid metabolism that is associated with worsening fibrosis and liver injury. Experimental animal studies have demonstrated that enteric but not colonic dysbiosis was associated with hepatobiliary inflammation, and small bowel bacterial overgrowth in rats resulted in hepatobiliary inflammation resembling histological and cholangiography features of PSC.

Human studies have revealed that increased abundance of Escherichia and Megasphaera and lower levels of Prevotella,
Roseburia and Bacteroides were associated with PSC and inflammatory bowel disease. ${ }^{68}$ Even though a single-case study, done longitudinally over 12 months, Philips et al. ${ }^{69}$ demonstrated that endoscopic FMT ( $250 \mathrm{~mL}$, distal duodenum) repeated weekly for 4-weeks improved symptoms, liver biochemistry, bile acid fractions and survival in tandem with beneficial changes in bacterial communities and functional metabolites in a patient with advanced PSC and recurrentcholangitis listed for liver transplantation (Fig. 3). Allegretti et al. ${ }^{70}$ performed the first pilot study on FMT in 10 patients with PSC, of whom 9 had ulcerative colitis, and 1 had Crohn's colitis. The mean baseline alkaline phosphatase level was 489 $\mathrm{U} / \mathrm{L}$. Overall, $30 \%$ experienced $\mathrm{a} \geq 50 \%$ decrease in alkaline phosphatase levels post-FMT. The bacterial diversity increased in all patients post-FMT, in the first week itself and abundance of engrafted microbial communities after FMT also correlated with the decrease in alkaline phosphatase levels.

Functional and compositional changes of GM have been demonstrated in patients with chronic hepatitis B virus infection-related cirrhosis, in the form of reduced abundance of Bifidobacteria and Lactobacillus, and high levels of Enterococcus. In another study, it was shown that, in hepatitis B virus-related cirrhosis reduction in Bacteroidetes and increased levels of Proteobacteria compared to the healthy group was notable. Healthy donor FMT, in addition to standard antivirals, was shown to be significantly more effective in clearance of the hepatitis $\mathrm{B}$ e antigen when compared to antiviral therapy alone. ${ }^{71,72}$

In patients with hepatitis C virus (HCV)-related cirrhosis, the microbial diversity was found to be lower when compared to healthy controls. HCV can alter the GM through 
Philips C.A. et al: Intestinal microbiota reinstitution therapy

Table 1. Summary of association of gut microbiota in various liver diseases

\begin{tabular}{|c|c|c|c|}
\hline Disease & \multicolumn{2}{|c|}{ Pertinent associated microbiota and metabolites } & Comments \\
\hline \multirow[t]{2}{*}{ NAFL } & Increase & $\begin{array}{l}\text { Blautia, Dorea, Streptococcus, Clostridium } \\
\text { Butanoic acid, Propanoic acid, Phenylacetic acid, } \\
\text { Isobutyric acid, Unconjugated cholic acid, Ethanol }\end{array}$ & \multirow[t]{16}{*}{$\begin{array}{l}\text { In animal models, reversing microbiota } \\
\text { changes reversed hepatic steatosis in } \\
\text { the absence of weight loss }\end{array}$} \\
\hline & Decrease & $\begin{array}{l}\text { Oscillospira, Coprococcus, Fecalibacterium } \\
\text { 2-butanone }\end{array}$ & \\
\hline \multirow[t]{2}{*}{ NASH } & Increase & $\begin{array}{l}\text { Escherichia, Blautia, Dorea, Lactobacillus, } \\
\text { Clostridium, Allisonella, Bacteroides } \\
\text { Chenodeoxycholic acid, Unconjugated cholate, } \\
\text { Lithocholic acid, Ethanol, 4-Methyl-2-pentanone }\end{array}$ & \\
\hline & Decrease & Oscillospira, Coprococcus, Fecalibacterium & \\
\hline \multirow[t]{2}{*}{$\begin{array}{l}\text { NASH-related } \\
\text { advanced } \\
\text { fibrosis }\end{array}$} & Increase & $\begin{array}{l}\text { Blautia, Roseburia, Streptococcus, Lactobacillus, } \\
\text { Enterococcus, Bacteroides, Escherichia, Klebsiella } \\
\text { 3-Phenylpropanoate, 3-4-Hydroxyphenyl-lactate }\end{array}$ & \\
\hline & Decrease & Ruminococcus, Akkermansia & \\
\hline \multirow{2}{*}{$\begin{array}{l}\text { NAFLD-related } \\
\text { HCC }\end{array}$} & Increase & Enterococcus, Oscillospira, Bacteroides & \\
\hline & Decrease & Blautia, Bifidobacterium & \\
\hline \multirow{2}{*}{$\begin{array}{l}\text { NASH in obese } \\
\text { children }\end{array}$} & Increase & Prevotella, Escherichia coli & \\
\hline & Decrease & Bifidobacterium, Alistipes, Blautia & \\
\hline \multirow[t]{2}{*}{$\begin{array}{l}\text { Alcoholic liver } \\
\text { disease without } \\
\text { cirrhosis }\end{array}$} & Increase & $\begin{array}{l}\text { Proteobacteria } \\
\text { Threonine, Glutamine, Guanidino-succinate, } \\
\text { Propionate, Isobutyrate, Dimethyl disulfide, } \\
\text { Dimethyl trisulfide, Urinary 3- } \\
\text { hydroxytetradecanedioic acid, and so-citric acid }\end{array}$ & \\
\hline & Decrease & $\begin{array}{l}\text { Bacteroidetes, Ruminococcaceae } \\
\text { Urinary sebacic acid }\end{array}$ & \\
\hline \multirow{2}{*}{$\begin{array}{l}\text { Alcoholic } \\
\text { cirrhosis with } \\
\text { abstinence }\end{array}$} & Increase & Enterobacteriaceae & \\
\hline & Decrease & Lachnospiraceae and Ruminococcaceae & \\
\hline \multirow{2}{*}{$\begin{array}{l}\text { Alcoholic } \\
\text { cirrhosis with } \\
\text { active drinking }\end{array}$} & Increase & Oral-origin microbiota and Lactobacillaceae & \\
\hline & Decrease & $\begin{array}{l}\text { Citrate, Malate, Phosphate, Threonine, Ornithine, } \\
\text { Serine, Ribosine, Orotic acid, Hexanoate }\end{array}$ & \\
\hline \multirow[t]{2}{*}{$\begin{array}{l}\text { Alcoholic } \\
\text { hepatitis }\end{array}$} & Increase & $\begin{array}{l}\text { Enterobacteriaceae, Streptococcaceae, } \\
\text { Actinobacterium, Bifidobacterium, Fusobacteria } \\
\text { Eicosapentaenoate, Docosapentaenoate, Benzoic } \\
\text { acid metabolites }\end{array}$ & \multirow{2}{*}{$\begin{array}{l}\text { Higher total serum bilirubin in patients } \\
\text { with higher fecal abundance of } \\
\text { Enterobacteria Lower total serum } \\
\text { bilirubin in patients with higher fecal } \\
\text { abundance of Clostridiales } \\
\text { Akkermansia muciniphila abundance } \\
\text { reduced with increasing } \\
\text { severity of alcoholic liver disease; } \\
\text { lowest in alcoholic hepatitis }\end{array}$} \\
\hline & Decrease & $\begin{array}{l}\text { Akkermansia muciniphila } \\
\text { Monoacylglycerols, Malate, Fumarate, Citrate, } \\
\text { Glycodeoxycholate }\end{array}$ & \\
\hline \multirow[t]{2}{*}{$\begin{array}{l}\text { Cirrhosis (any } \\
\text { etiology) }\end{array}$} & Increase & $\begin{array}{l}\text { Proteobacteria, Fusobacteria, Clostridium } \\
\text { clusters XI } \\
\text { Enterobacteriaceae, Streptococcaceae, } \\
\text { Leuconostocaceae, Lactobacillaceae, } \\
\text { Alcaligenaceae } \\
\text { Acidaminococcus, Enterococcus, Burkholderia, } \\
\text { Ralstonia } \\
\text { Proteus }\end{array}$ & \multirow[t]{2}{*}{$\begin{array}{l}\text { Reduction in levels of Lachnospira and } \\
\text { increase in level of Streptococcus } \\
\text { associated with higher Child-Pugh } \\
\text { scores } \\
\text { Enterobacterium associated with } \\
\text { spontaneous bacterial peritonitis } \\
\text { Bacteroidaceaeae and Clostridiales XIV } \\
\text { predictors of } 90 \text {-day hospitalization } \\
\text { and higher Child-Pugh and MELD } \\
\text { scores }\end{array}$} \\
\hline & Decrease & $\begin{array}{l}\text { Bacteroidetes } \\
\text { Lachnospiraceae, Ruminococcaceae } \\
\text { Clostridium-Incertae sedis - XIV } \\
\text { Dorea, Subdoligranumum }\end{array}$ & \\
\hline
\end{tabular}


Table 1. (continued)

\begin{tabular}{|c|c|c|c|}
\hline Disease & \multicolumn{2}{|c|}{ Pertinent associated microbiota and metabolites } & Comments \\
\hline \multirow[t]{2}{*}{$\begin{array}{l}\text { Acute-on- } \\
\text { chronic liver } \\
\text { failure }\end{array}$} & $\begin{array}{l}\text { Predictors } \\
\text { of poor } \\
\text { outcomes }\end{array}$ & $\begin{array}{l}\text { Enterobacteriaceae, Campylobacteriaceae, } \\
\text { Pasteurellaceae, Enterococcaceae, } \\
\text { Streptococcaceae }\end{array}$ & \\
\hline & $\begin{array}{l}\text { Reduction } \\
\text { in poor } \\
\text { outcomes }\end{array}$ & Lachnospiraceae, Clostridiales & \\
\hline \multirow[t]{2}{*}{$\begin{array}{l}\text { Hepatic } \\
\text { encephalopathy }\end{array}$} & Increase & $\begin{array}{l}\text { Alcaligenaceae, Porphyromonadaceae } \\
\text { Veillonella, Megasphaera, Bifidobacterium, } \\
\text { Enterococcus, Streptococcus salivarius }\end{array}$ & \\
\hline & Decrease & Roseburia & \\
\hline \multirow[t]{2}{*}{$\mathrm{HCC}$} & Increase & $\begin{array}{l}\text { Escherichia coli, Escherichia-Shigella, } \\
\text { Enterococcus, Proteus, Veillonella, } \\
\text { Actinobacterium, Gemmiger }\end{array}$ & \\
\hline & Decrease & $\begin{array}{l}\text { Fecalibacterium, Rumonococcus, } \\
\text { Ruminoclostridium Pseudobutyrivibrio, } \\
\text { Lachnoclostridium Phascolarctobacterium, } \\
\text { Parabacteroides }\end{array}$ & \\
\hline \multirow{2}{*}{$\begin{array}{l}\text { Chronic } \\
\text { hepatitis B } \\
\text { virus-related } \\
\text { cirrhosis }\end{array}$} & Increase & Proteobacteria, Enterococcus & \\
\hline & Decrease & Bifidobacterium, Lactobacillus & \\
\hline \multirow{2}{*}{$\begin{array}{l}\text { Chronic } \\
\text { hepatitis C } \\
\text { virus-related } \\
\text { cirrhosis }\end{array}$} & Increase & Prevotella, Fecalibacterium & \\
\hline & Decrease & Acinetobacter, Veillonella, Phascolarctobacterium & \\
\hline \multirow{2}{*}{$\begin{array}{l}\text { Autoimmune } \\
\text { hepatitis }\end{array}$} & Increase & Veillonella dispar & \multirow{2}{*}{$\begin{array}{l}\text { V. dispar associated with elevation of } \\
\text { aspartate aminotransferase levels and } \\
\text { severity of autoimmune hepatitis }\end{array}$} \\
\hline & Decrease & Bifidobacterium, Lactobacillus & \\
\hline \multirow{2}{*}{$\begin{array}{l}\text { Primary } \\
\text { sclerosing } \\
\text { cholangitis }\end{array}$} & Increase & $\begin{array}{l}\text { Barnesiellaceae, Lachnospiraceae } \\
\text { Blautia, Escherichia, Ruminococcus, Megasphaera }\end{array}$ & \multirow{2}{*}{$\begin{array}{l}\text { Additionally, increased proportion of } \\
\text { fungi Exophiala and a decreased } \\
\text { proportion of Saccharomyces } \\
\text { cerevisiae notable in patients with } \\
\text { primary sclerosing cholangitis and } \\
\text { inflammatory bowel disease }\end{array}$} \\
\hline & Decrease & $\begin{array}{l}\text { Uncultured Clostridiales II } \\
\text { Prevotella, Roseburia, Bacteroides }\end{array}$ & \\
\hline $\begin{array}{l}\text { Drug-induced } \\
\text { liver injury }\end{array}$ & \multicolumn{2}{|c|}{$\begin{array}{c}\text { Increase in Mucispirillum, Turicibacter and Ruminococcus } \\
\text { associated with higher risk of toxicity to acetaminophen } \\
\text { Metabolite 1-phenyl-1,2-propanedione associated with diurnal } \\
\text { variation of acetaminophen induced hepatotoxicity }\end{array}$} & \\
\hline $\begin{array}{l}\text { Post-liver } \\
\text { transplantation }\end{array}$ & \multicolumn{2}{|c|}{$\begin{array}{l}\text { Higher fecal levels of Klebsiella, Escherichia, Shigella in post- } \\
\text { transplantation period associated with infections }\end{array}$} & $\begin{array}{l}\text { Fecal microbiome index consisting of } \\
\text { Staphylococcus and Prevotella useful in } \\
\text { identifying patients post-liver } \\
\text { transplant who develop abnormal liver } \\
\text { tests }\end{array}$ \\
\hline
\end{tabular}

* Pertinent metabolites associated with prominent bacterial communities in the given liver disease condition

Abbreviations: HCC, hepatocellular carcinoma; NAFL, nonalcoholic fatty liver; NAFLD, nonalcoholic fatty liver disease; NASH, nonalcoholic steatohepatitis.

immunoglobulin A produced by infected B-lymphocytes. In the GM of Egyptian patients with HCV, higher abundance of Prevotella and Faecalibacterium and lower levels of Acinetobacter, Veillonella and Phascolarctobacterium were notable. The role of Prevotella or Faecalibacterium to Bifidobacterium ratio has been demonstrated as a biomarker for fibrosis progression in HCV-infected patients. However, in patients with HCV-related cirrhosis, gut dysbiosis can persist, regardless of long-term sustained viral response. ${ }^{73}$
The intestinal microbiota influences drug and xenobiotic metabolisms, that can affect drug efficacy and toxicity. Microbiota-related drug metabolism is important for activation of some prodrugs. ${ }^{74}$ The GM also takes part in additional metabolic reactions associated with some drugs, such as acetylation, decarboxylation, dihydroxylation and demethylation. Microbiota-derived metabolites can indirectly affect xenobiotic metabolism pathways. It was demonstrated that the intestinal microbiota modulated susceptibility to 
acetaminophen (APAP)-induced acute liver injury. The relative abundance of Mucispirillum, Turicibacter and Ruminococcus before APAP dosing was found to be associated with increased hepatotoxicity. ${ }^{75}$

APAP-induced liver injury has diurnal variation. APAP causes more hepatotoxicity during consumption at night compared to morning. It was demonstrated that the gut microbial metabolite, 1-phenyl-1,2-propanedione was involved in the rhythmic hepatotoxicity induced by APAP, by depleting hepatic glutathione levels. The anti-inflammatory drug, salicylazosulfapyridine, underwent degradation in conventional rats and when cultured with human gut bacteria but not in germ-free rats, demonstrating the role of GM in drug transformations. Wang et al. $^{76}$ showed that healthy donor FMT prevented HE in rats with carbon tetrachloride-induced acute liver failure.

The intestinal microbiota has been shown to play a central role in sensitization of sepsis-induced liver injury, and microbiota-associated granisetron production resulted in amelioration of liver injury during sepsis development in a mouse model of cecal ligation puncture. ${ }^{77}$

A recent case series showcased beneficial outcomes with FMT-related reconstitution of the gut microbiome on immune checkpoint inhibitors in colitis associated with a relative increase in regulatory T-cells within the colonic mucosa. ${ }^{78}$

Lu et al. ${ }^{79}$ showed that there was a higher relative abundance of Klebsiella, Escherichia and Shigella and reduced levels of beneficial butyrate-producing bacteria among liver transplant recipients. The authors established a fecal microbiome index (specific alterations in Staphylococcus and Prevotella) that could be used for assessing liver recipients at risk of liver dysfunction. A summary of pertinent GM association with various liver diseases is shown in Table 1.

\section{Conclusions}

GM plays a central role in the initiation and progression of certain liver diseases and changes in GM drive the pathophysiology of select liver diseases. There are robust data on the role of GM in the development of NAFLD and NASH, severe $\mathrm{AH}$, complications of cirrhosis and especially HE and PSC. High-quality studies have also shown specific roles played by the GM in the progression of diseases such as drug-induced liver injury, AIH and chronic viral hepatitis. The benefits of therapeutic modulation of GM in liver diseases such as $\mathrm{AH}, \mathrm{HE}$ and PSC are well documented but high-quality randomized trials are lacking. The future of liver disease management could well include microbiota modulation based on the highquality bench-to-bedside research in the coming years.

\section{Funding}

None to declare.

\section{Conflict of interest}

CAP receives advisory fees and research grant support from Cipla $^{\circledR}$ and Samarth Lifesciences ${ }^{\circledR}$. The other authors have no conflict of interests related to this publication.

\section{Author contributions}

Designing the research study (CAP, PA, PKY, GNR), collecting the data (CAP, RA, SR, TG, SK), writing the manuscript (CAP,
$\mathrm{PA}, \mathrm{SR}, \mathrm{RA})$, critically reviewing and revising the manuscript (CAP, PA, PKY, GNR, RA, SR, TG, SK).

\section{References}

[1] Gutleben J, Chaib De Mares M, van Elsas JD, Smidt H, Overmann J, Sipkema D. The multi-omics promise in context: from sequence to microbial isolate. Crit Rev Microbiol 2018;44:212-229. doi: 10.1080/1040841X.2017.1332003.

[2] Zuñiga C, Zaramela L, Zengler K. Elucidation of complexity and prediction of interactions in microbial communities. Microb Biotechnol 2017;10:15001522. doi: 10.1111/1751-7915.12855.

[3] Fondi M, Liò P. Multi-omics and metabolic modelling pipelines: challenges and tools for systems microbiology. Microbiol Res 2015;171:52-64. doi: 10. 1016/j.micres.2015.01.003.

[4] Abram F. Systems-based approaches to unravel multi-species microbial community functioning. Comput Struct Biotechnol J 2014;13:24-32. doi: 10. 1016/j.csbj.2014.11.009.

[5] Cani PD. Human gut microbiome: hopes, threats and promises. Gut 2018; 67:1716-1725. doi: 10.1136/gutjnl-2018-316723.

[6] Mohajeri MH, Brummer RJM, Rastall RA, Weersma RK, Harmsen HJM, Faas M, et al. The role of the microbiome for human health: from basic science to clinical applications. Eur J Nutr 2018;57:1-14. doi: 10.1007/s00394-0181703-4.

[7] Goodrich JK, Davenport ER, Beaumont M, Jackson MA, Knight R, Ober C, et al. Genetic determinants of the gut microbiome in UK twins. Cell Host Microbe 2016;19:731-743. doi: 10.1016/j.chom.2016.04.017.

[8] Liang D, Leung RK, Guan W, Au WW. Involvement of gut microbiome in human health and disease: brief overview, knowledge gaps and research opportunities. Gut Pathog 2018;10:3. doi: 10.1186/s13099-018-0230-4.

[9] Dieterich W, Schink M, Zopf Y. Microbiota in the gastrointestinal tract. Med Sci (Basel) 2018;6:116. doi: 10.3390/medsci6040116

[10] Dominguez-Bello MG, Godoy-Vitorino F, Knight R, Blaser MJ. Role of the microbiome in human development. Gut 2019;68:1108-1114. doi: 10 . 1136/gutjnl-2018-317503.

[11] Gordo I. Evolutionary change in the human gut microbiome: From a static to a dynamic view. PLoS Biol 2019;17:e3000126. doi: 10.1371/journal.pbio. 3000126.

[12] Thursby E, Juge N. Introduction to the human gut microbiota. Biochem J 2017;474:1823-1836. doi: 10.1042/BCJ20160510.

[13] Yang JY, Kweon MN. The gut microbiota: a key regulator of metabolic diseases. BMB Rep 2016;49:536-541. doi: 10.5483/bmbrep.2016.49.10.144.

[14] Gomes AC, Hoffmann C, Mota JF. The human gut microbiota: Metabolism and perspective in obesity. Gut Microbes 2018;9:308-325. doi: 10. 1080/19490976.2018.1465157.

[15] Lamichhane S, Sen P, Dickens AM, Orešič M, Bertram HC. Gut metabolome meets microbiome: A methodological perspective to understand the relationship between host and microbe. Methods 2018;149:3-12. doi: 10 . 1016/j.ymeth.2018.04.029.

[16] Nagpal R, Kumar M, Yadav AK, Hemalatha R, Yadav H, Marotta F, et al. Gut microbiota in health and disease: an overview focused on metabolic inflammation. Benef Microbes 2016;7:181-194. doi: 10.3920/bm2015.0062.

[17] Boulangé CL, Neves AL, Chilloux J, Nicholson JK, Dumas ME. Impact of the gut microbiota on inflammation, obesity, and metabolic disease. Genome Med 2016;8:42. doi: 10.1186/s13073-016-0303-2.

[18] Janssen AW, Kersten S. Potential mediators linking gut bacteria to metabolic health: a critical view. J Physiol 2017;595:477-487. doi: 10.1113/JP272476.

[19] Ghazalpour A, Cespedes I, Bennett B], Allayee H. Expanding role of gut microbiota in lipid metabolism. Curr Opin Lipidol 2016;27:141-147. doi: 10.1097/MOL.0000000000000278.

[20] Diether NE, Willing BP. Microbial fermentation of dietary protein: An important factor in diet-microbe-host interaction. Microorganisms 2019;7:19. doi: 10.3390/microorganisms7010019.

[21] Bik EM, Ugalde JA, Cousins J, Goddard AD, Richman J, Apte ZS. Microbial biotransformations in the human distal gut. Br J Pharmacol 2018;175:44044414. doi: $10.1111 / \mathrm{bph} .14085$

[22] Madsen L, Myrmel LS, Fjære E, Liaset B, Kristiansen K. Links between dietary protein sources, the gut microbiota, and obesity. Front Physiol 2017;8:1047. doi: 10.3389/fphys.2017.01047.

[23] Cani PD, Amar J, Iglesias MA, Poggi M, Knauf C, Bastelica D, et al. Metabolic endotoxemia initiates obesity and insulin resistance. Diabetes 2007;56: 1761-1772. doi: $10.2337 / \mathrm{db06}-1491$.

[24] Giannelli V, Di Gregorio V, Iebba V, Giusto M, Schippa S, Merli M, et al. Microbiota and the gut-liver axis: bacterial translocation, inflammation and infection in cirrhosis. World J Gastroenterol 2014;20:16795-16810. doi: 10. 3748/wjg.v20.i45.16795.

[25] Fukui H. Gut-liver axis in liver cirrhosis: How to manage leaky gut and endotoxemia. World J Hepatol 2015;7:425-442. doi: 10.4254/wjh.v7.i3.425. 
[26] Usami M, Miyoshi M, Yamashita H. Gut microbiota and host metabolism in liver cirrhosis. World J Gastroenterol 2015;21:11597-11608. doi: 10. 3748/wjg.v21.i41.11597.

[27] Patel VC, White H, Støy S, Bajaj JS, Shawcross DL. Clinical science workshop: targeting the gut-liver-brain axis. Metab Brain Dis 2016;31:13271337. doi: 10.1007/s11011-015-9743-4.

[28] Wiest R, Albillos A, Trauner M, Bajaj JS, Jalan R. Targeting the gut-liver axis in liver disease. J Hepatol 2017;67:1084-1103. doi: 10.1016/j.jhep.2017.05. 007.

[29] Le Roy T, Llopis M, Lepage P, Bruneau A, Rabot S, Bevilacqua C, et al. Intestinal microbiota determines development of non-alcoholic fatty liver disease in mice. Gut 2013;62:1787-1794. doi: 10.1136/gutjnl-2012-303816.

[30] Tripathi A, Debelius J, Brenner DA, Karin M, Loomba R, Schnabl B, et al. The gut-liver axis and the intersection with the microbiome. Nat Rev Gastroenterol Hepatol 2018;15:397-411. doi: 10.1038/s41575-018-0011-z.

[31] de Faria Ghetti F, Oliveira DG, de Oliveira JM, de Castro Ferreira LEVV, Cesar $\mathrm{DE}$, Moreira APB. Influence of gut microbiota on the development and progression of nonalcoholic steatohepatitis. Eur J Nutr 2018;57:861-876. doi: 10.1007/s00394-017-1524-x.

[32] de Groot PF, Frissen MN, de Clercq NC, Nieuwdorp M. Fecal microbiota transplantation in metabolic syndrome: History, present and future. Gut Microbes 2017;8:253-267. doi: 10.1080/19490976.2017.1293224.

[33] Behrouz V, Jazayeri S, Aryaeian N, Zahedi MJ, Hosseini F. Effects of probiotic and prebiotic supplementation on leptin, adiponectin, and glycemic parameters in non-alcoholic fatty liver disease: A randomized clinical trial. Middle East J Dig Dis 2017;9:150-157. doi: 10.15171/mejdd.2017.66.

[34] Kolodziejczyk AA, Zheng D, Shibolet O, Elinav E. The role of the microbiome in NAFLD and NASH. EMBO Mol Med 2019;11:e9302. doi: 10.15252/emmm. 201809302.

[35] Lynch SV, Pedersen O. The human intestinal microbiome in health and disease. N Engl J Med 2016;375:2369-2379. doi: 10.1056/NEJMra1600266.

[36] Smith PM, Howitt MR, Panikov N, Michaud M, Gallini CA, Bohlooly-Y M, et al. The microbial metabolites, short-chain fatty acids, regulate colonic Treg cell homeostasis. Science 2013;341:569-573. doi: 10.1126/science.1241165.

[37] Cammarota G, Ianiro G, Tilg H, Rajilić-Stojanović M, Kump P, Satokari R, et al. European consensus conference on faecal microbiota transplantation in clinical practice. Gut 2017;66:569-580. doi: 10.1136/gutjnl-2016313017.

[38] Dong TS, Jacobs JP. Nonalcoholic fatty liver disease and the gut microbiome: Are bacteria responsible for fatty liver? Exp Biol Med (Maywood) 2019;244: 408-418. doi: 10.1177/1535370219836739.

[39] Sharpton SR, Ajmera V, Loomba R. Emerging role of the gut microbiome in nonalcoholic fatty liver disease: From composition to function. Clin Gastroenterol Hepatol 2019;17:296-306. doi: 10.1016/j.cgh.2018.08.065.

[40] Sáez-Lara MJ, Robles-Sanchez C, Ruiz-Ojeda FJ, Plaza-Diaz J, Gil A. Effects of probiotics and synbiotics on obesity, insulin resistance syndrome, type 2 diabetes and non-alcoholic fatty liver disease: A review of human clinical trials. Int J Mol Sci 2016;17:928. doi: 10.3390/ijms17060928.

[41] Ma YY, Li L, Yu CH, Shen Z, Chen LH, Li YM. Effects of probiotics on nonalcoholic fatty liver disease: a meta-analysis. World J Gastroenterol 2013; 19:6911-6918. doi: 10.3748/wjg.v19.i40.6911.

[42] Khoruts A, Brandt LJ. Fecal microbiota transplant: A rose by any other name. Am J Gastroenterol 2019;114:1176. doi: 10.14309/ajg.0000000000000286.

[43] Bajaj JS, Hays RA. Manipulation of the gut-liver axis using microbiome restoration therapy in primary sclerosing cholangitis. Am J Gastroenterol 2019; 114:1027-1029. doi: 10.14309/ajg.0000000000000191.

[44] Zhou D, Pan Q, Shen F, Cao HX, Ding WJ, Chen YW, et al. Total fecal microbiota transplantation alleviates high-fat diet-induced steatohepatitis in mice via beneficial regulation of gut microbiota. Sci Rep 2017;7:1529. doi: 10 . 1038/s41598-017-01751-y.

[45] Vrieze A, Van Nood E, Holleman F, Salojärvi J, Kootte RS, Bartelsman JF, et al. Transfer of intestinal microbiota from lean donors increases insulin sensitivity in individuals with metabolic syndrome. Gastroenterology 2012;143: 913-916.e7. doi: 10.1053/j.gastro.2012.06.031.

[46] Xue LF, Luo WH, Wu LH, He XX, Xia HHX, Chen Y. Fecal microbiota transplantation for the treatment of nonalcoholic fatty liver disease. Explor Res Hypothesis Med 2019;4:12. doi: 10.14218/ERHM.2018.00025.

[47] Llopis M, Cassard AM, Wrzosek L, Boschat L, Bruneau A, Ferrere G, et al. Intestinal microbiota contributes to individual susceptibility to alcoholic liver disease. Gut 2016;65:830-839. doi: 10.1136/gutjnl-2015-310585.

[48] Philips CA, Pande A, Shasthry SM, Jamwal KD, Khillan V, Chandel SS, et al. Healthy donor fecal microbiota transplantation in steroid-ineligible severe alcoholic hepatitis: A pilot study. Clin Gastroenterol Hepatol 2017;15:600602. doi: 10.1016/j.cgh.2016.10.029.

[49] Scarpellini E, Forlino M, Lupo M, Rasetti C, Fava G, Abenavoli L, et al. Gut microbiota and alcoholic liver disease. Rev Recent Clin Trials 2016;11:213219. doi: 10.2174/1574887111666160810100538.

[50] Grander C, Adolph TE, Wieser V, Lowe P, Wrzosek L, Gyongyosi B, et al. Recovery of ethanol-induced Akkermansia muciniphila depletion ameliorates alcoholic liver disease. Gut 2018;67:891-901. doi: 10.1136/gutjnl-2016313432.

[51] Ciocan D, Voican CS, Wrzosek L, Hugot C, Rainteau D, Humbert L, et al. Bile acid homeostasis and intestinal dysbiosis in alcoholic hepatitis. Aliment Pharmacol Ther 2018;48:961-974. doi: 10.1111/apt.14949.

[52] Puri P, Liangpunsakul S, Christensen JE, Shah VH, Kamath PS, Gores G], et al. The circulating microbiome signature and inferred functional metagenomics in alcoholic hepatitis. Hepatology 2018;67:1284-1302. doi: 10. 1002/hep.29623.

[53] Bluemel S, Wang L, Kuelbs C, Moncera K, Torralba M, Singh H, et al. Intestinal and hepatic microbiota changes associated with chronic ethanol administration in mice. Gut Microbes 2019. doi: 10.1080/19490976.2019. 1595300.

[54] Kirpich IA, Solovieva NV, Leikhter SN, Shidakova NA, Lebedeva OV, Sidorov PI, et al. Probiotics restore bowel flora and improve liver enzymes in human alcohol-induced liver injury: a pilot study. Alcohol 2008;42:675-682. doi: 10.1016/j.alcohol.2008.08.006.

[55] Han SH, Suk KT, Kim DJ, Kim MY, Baik SK, Kim YD, et al. Effects of probiotics (cultured Lactobacillus subtilis/Streptococcus faecium) in the treatment of alcoholic hepatitis: randomized-controlled multicenter study. Eur ] Gastroenterol Hepatol 2015;27:1300-1306. doi: 10.1097/MEG.0000000000000458.

[56] Philips CA, Pande A, Shasthry SM, Jamwal KD, Khillan V, Chandel SS, et al. Healthy donor fecal microbiota transplantation in steroid-ineligible severe alcoholic hepatitis: A pilot study. Clin Gastroenterol Hepatol 2017;15:600602. doi: 10.1016/j.cgh.2016.10.029.

[57] Philips CA, Phadke N, Ganesan K, Ranade S, Augustine P. Corticosteroids, nutrition, pentoxifylline, or fecal microbiota transplantation for severe alcoholic hepatitis. Indian J Gastroenterol 2018;37:215-225. doi: 10. 1007/s12664-018-0859-4.

[58] Fukui H. Gut microbiome-based therapeutics in liver cirrhosis: Basic consideration for the next step. J Clin Transl Hepatol 2017;5:249-260. doi: 10. 14218/JCTH.2017.00008.

[59] Hartmann P, Chu H, Duan Y, Schnabl B. Gut microbiota in liver disease: too much is harmful, nothing at all is not helpful either. Am J Physiol Gastrointest Liver Physiol 2019;316:G563-G573. doi: 10.1152/ajpgi.00370.2018.

[60] Bajaj JS. The role of microbiota in hepatic encephalopathy. Gut Microbes 2014;5:397-403. doi: 10.4161/gmic.28684.

[61] Bajaj JS, Vargas HE, Reddy KR, Lai JC, O'Leary JG, Tandon P, et al. Association between intestinal microbiota collected at hospital admission and outcomes of patients with cirrhosis. Clin Gastroenterol Hepatol 2019;17: 756-765.e3. doi: 10.1016/j.cgh.2018.07.022.

[62] Bajaj JS, Kassam Z, Fagan A, Gavis EA, Liu E, Cox IJ, et al. Fecal microbiota transplant from a rational stool donor improves hepatic encephalopathy: $A$ randomized clinical trial. Hepatology 2017;66:1727-1738. doi: 10 . 1002/hep.29306.

[63] Bajaj JS, Salzman NH, Acharya C, Sterling RK, White MB, Gavis EA, et al. Fecal microbial transplant capsules are safe in hepatic encephalopathy: A phase 1 , randomized, placebo-controlled trial. Hepatology 2019;70:16901703. doi: $10.1002 /$ hep.30690.

[64] Sanduzzi Zamparelli M, Rocco A, Compare D, Nardone G. The gut microbiota: A new potential driving force in liver cirrhosis and hepatocellular carcinoma. United European Gastroenterol J 2017;5:944-953. doi: 10. $1177 / 2050640617705576$

[65] Wang L, Wan YJY. The role of gut microbiota in liver disease development and treatment. Liver Research 2019;3:3-18. doi: 10.1016/j.livres.2019.02.001.

[66] Czaja AJ. Factoring the intestinal microbiome into the pathogenesis of autoimmune hepatitis. World J Gastroenterol 2016;22:9257-9278. doi: 10. 3748/wjg.v22.i42.9257.

[67] Wei Y, Li Y, Yan L, Sun C, Miao Q, Wang Q, et al. Alterations of gut microbiome in autoimmune hepatitis. Gut 2019. doi: 10.1136/gutjnl-2018-317836.

[68] Hov JR, Kummen M. Intestinal microbiota in primary sclerosing cholangitis. Curr Opin Gastroenterol 2017;33:85-92. doi: 10.1097/MOG.0000000000000334.

[69] Philips CA, Augustine P, Phadke N. Healthy donor fecal microbiota transplantation for recurrent bacterial cholangitis in primary sclerosing cholangitis - A single case report. J Clin Transl Hepatol 2018;6:438-441. doi: 10. 14218/JCTH.2018.00033.

[70] Allegretti JR, Kassam Z, Carrellas M, Mullish BH, Marchesi JR, Pechlivanis A, et al. Fecal microbiota transplantation in patients with primary sclerosing cholangitis: A pilot clinical trial. Am J Gastroenterol 2019;114:1071-1079. doi: 10.14309/ajg.0000000000000115.

[71] Kang Y, Cai Y. Gut microbiota and hepatitis-B-virus-induced chronic liver disease: implications for faecal microbiota transplantation therapy. J Hosp Infect 2017;96:342-348. doi: 10.1016/j.jhin.2017.04.007.

[72] Ren YD, Ye ZS, Yang LZ, Jin LX, Wei WJ, Deng YY, et al. Fecal microbiota transplantation induces hepatitis $B$ virus e-antigen ( $\mathrm{HBeAg}$ ) clearance in patients with positive $\mathrm{HBeAg}$ after long-term antiviral therapy. Hepatology 2017;65:1765-1768. doi: 10.1002/hep.29008.

[73] Aly AM, Adel A, El-Gendy AO, Essam TM, Aziz RK. Gut microbiome alterations in patients with stage 4 hepatitis C. Gut Pathog 2016;8:42. doi: 10. 1186/s13099-016-0124-2. 
[74] Wilson ID, Nicholson JK. Gut microbiome interactions with drug metabolism efficacy, and toxicity. Transl Res 2017;179:204-222. doi: 10.1016/j.trsl. 2016.08.002.

[75] Gong S, Lan T, Zeng L, Luo H, Yang X, Li N, et al. Gut microbiota mediates diurnal variation of acetaminophen induced acute liver injury in mice. J Hepatol 2018;69:51-59. doi: 10.1016/j.jhep.2018.02.024.

[76] Wang WW, Zhang Y, Huang XB, You N, Zheng L, Li J. Fecal microbiota transplantation prevents hepatic encephalopathy in rats with carbon tetrachloride-induced acute hepatic dysfunction. World J Gastroenterol 2017;23: 6983-6994. doi: 10.3748/wjg.v23.i38.6983.
[77] Gong S, Yan Z, Liu Z, Niu M, Fang $\mathrm{H}$, Li N, et al. Intestinal microbiota mediates the susceptibility to polymicrobial sepsis-induced liver injury by granisetron generation in mice. Hepatology 2019;69:1751-1767. doi: 10.1002/hep.30361.

[78] Wang Y, Wiesnoski DH, Helmink BA, Gopalakrishnan V, Choi K, DuPont HL, et al. Fecal microbiota transplantation for refractory immune checkpoint inhibitor-associated colitis. Nat Med 2018;24:1804-1808. doi: 10.1038/s41591018-0238-9.

[79] Lu HF, Ren ZG, Li A, Zhang H, Xu SY, Jiang JW, et al. Fecal microbiome data distinguish liver recipients with normal and abnormal liver function from healthy controls. Front Microbiol 2019;10:1518. doi: 10.3389/fmicb.2019.01518. 